\title{
Elastic modulus and structural evolution of diamond-like carbon films deposited by RF-PACVD
}

\author{
Jin-Won Chunga,b, Dae-Hong Ko ${ }^{\mathrm{b}}$, Kwang Yong Eun ${ }^{\mathrm{a}}$, Kwang-Ryeol Lee ${ }^{\mathrm{a}, *}$ \\ ${ }^{a}$ Future Technology Research Division, Korea Institute of Science and Technology, P.O. Box 131, Cheongryang, Seoul, 130-650, South Korea \\ ${ }^{\mathrm{b}}$ Department of Ceramic Engineering, Yonsei University, Seoul, 120-701, South Korea
}

Received 7 August 2001; received in revised form 5 February 2002; accepted 6 February 2002

\begin{abstract}
Dependence of the elastic modulus of diamond-like carbon (DLC) films on film thickness was investigated by the recently suggested free overhang method. The DLC films of thickness ranging from 50 to $1300 \mathrm{~nm}$ were deposited on Si (100) wafer by 13.56- $\mathrm{MHz}$ radio frequency plasma assisted chemical vapor deposition (RF-PACVD) process. In the free overhang method, the elastic modulus of thin film could be measured without considering the substrate effect since the substrate was completely excluded from the measurement. It was observed that the elastic modulus of the very thin film was smaller than that of the thick film in both cases where the ion energy was higher and lower than the optimum value for dense and hard DLC film deposition. However, at the optimum ion energy, the elastic modulus was independent of film thickness. Raman spectroscopy analysis showed that the change of elastic modulus in very thin DLC films was intimately related to the structural evolution which occurred in the initial stage of the film deposition. When the ion energy was lower than the optimum value, more polymeric component was deposited in the initial stage. On the other hand, a more graphitic component was involved in very thin films at an ion energy higher than the optimum value. (c) 2002 Elsevier Science B.V. All rights reserved.
\end{abstract}

Keywords: Elastic modulus; Diamond-like carbon; Structural evolution; Free overhang method

\section{Introduction}

Many methods have been suggested to measure the mechanical properties of thin films. Nonoindentation is the most widely used method to measure the hardness and elastic modulus of thin films [1]. However, the difficulties of nanoindentation for very thin film arise from high sensitivity to the substrate, especially when applying to the system of large difference in mechanical properties between the substrate and the film. The substrate effect is more significant in measuring the elastic modulus than in measuring the hardness, because the elastic behavior during unloading is dominated by the elastic property of the substrate. (One should note that the elastic strain field is much wider than the plastic strain field.) Furthermore, the effect of truncated indenter tip should be carefully considered for the analysis of

\footnotetext{
*Corresponding author. Tel.: +82-2-958-5494; fax: +82-2-9585509.

E-mail address: krlee@kist.re.kr (K.-R. Lee).
}

shallow indentation [2]. Other methods using the propagation behavior of long wavelength acoustic phonon are also employed for elastic modulus measurement [35]. However, these methods need sophisticated instrumental and analytical techniques to be applied for very thin films. The difficulties also arise from the separation of the acoustic signal of the thin film from the mixed signal of the film and substrate. Therefore, precise measurement of the mechanical properties of extremely thin films remains a challenging problem.

Recently, Cho et al. suggested a simple method to measure the elastic modulus of diamond-like carbon (DLC) films which have a compressive residual stress $[6,7]$. The residual stress can be estimated from the curvature of the film/substrate composite. If one measures the strain of the film, which is required to adhere to the substrate, the biaxial elastic modulus can be obtained from a simple stress-strain relation of thin films. The suggested method involves chemical etching of the substrate for measurement of the strain. The etching process produces an unstressed DLC free over- 
hang or bridge of sinusoidal shape. By measuring the amplitude and wavelength of the sinusoidal deformation, the strain of the film could be obtained. The reliability of the free-overhang method was confirmed by comparing the measured elastic modulus with those obtained by nanoindentation when the film thickness was larger than $1 \mu \mathrm{m}$ [8]. Even if this method can be used only for films of compressive residual stress, the method has an important advantage for very thin films. Because the substrate is completely excluded from the measurement process, the substrate does not affect the measured value. The elastic modulus of very thin DLC film was measured by employing this method [8].

In DLC films, the mechanical properties of the film drastically vary with three-dimensional interlinks, which is enhanced by $\mathrm{sp}^{3}$ hybridization bonds [9]. Therefore, the characterization of the mechanical property is useful to help identify their chemical bond structure. In the previous work [8], we suggested that the structural evolution occurs during the initial stage of deposition when the DLC film was deposited by radio frequency plasma assisted chemical vapor deposition (RF-PACVD) method. This phenomenon would be significant for the industrial application of DLC films of thickness considerably smaller than $1 \mu \mathrm{m}$. For example, protective layers for hard disks of large storage capacity should be less than $5 \mathrm{~nm}$ due to the reduced magnetic spacing between the magnetic layer and the read/write head [10]. The mechanical property and atomic bond structure of the thin protective layer is one of the major concerns in the development of hard disks.

For more systematic investigations on the structural evolution of DLC films, we measured the elastic properties of very thin DLC films deposited by RF-PACVD. The films were deposited at various bias voltages of cathode and deposition pressures using methane as the precursor gas. Lee et al. reported the precursor gas effect on the structure of DLC film under similar deposition conditions [11]. The film deposited from methane exhibited a typical growth behavior depending on the ion energy; as the ion energy increased, the deposited film changed from polymeric amorphous carbon to dense diamond-like film, and then to graphitic type. Hence, films with a wide range of physical properties could be investigated in the present work. We observed that the degradation of mechanical properties in very thin films occurs either when the ion energy was too small resulting in a polymeric film, or when the ion energy was too large resulting in a graphitic type.

\section{Experimental}

DLC films were deposited on p-type Si (100) wafer by using capacitively coupled r.f. glow discharge of methane. Details of the experimental set-up were previously described [12]. The self-bias voltage of the

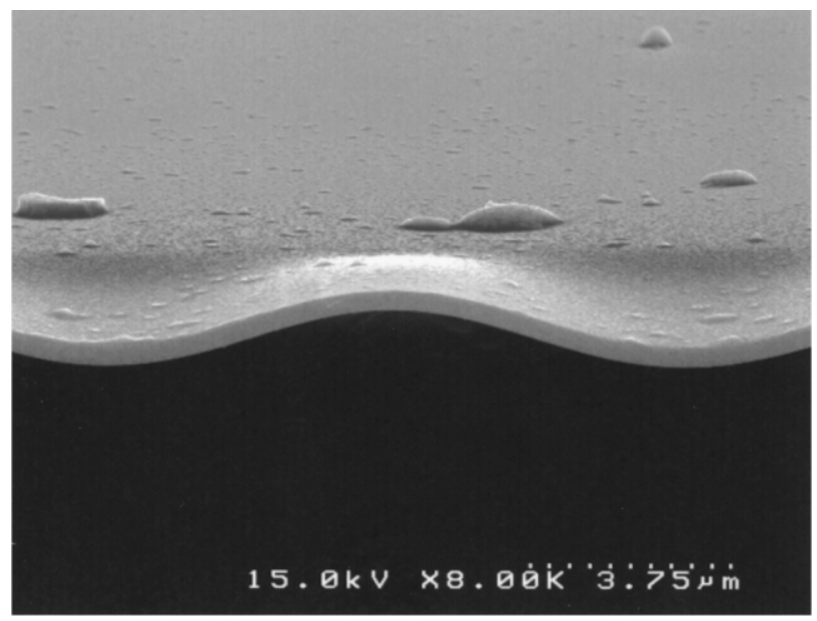

Fig. 1. Typical SEM microstructure of the DLC free-overhang.

cathode ranges from -100 to $-700 \mathrm{~V}$ by adjusting the supplied r.f. power. The deposition pressure, measured by a capacitance manometer, was varied from 9 to 25 mtorr. The film thickness was varied from 50 to 1300 $\mathrm{nm}$ by changing the deposition time. In order to measure the residual stress of the film, a thin $(100 \pm 5$ or $200 \pm 5$ $\mu \mathrm{m}$ in thickness) $\mathrm{Si}$ strip of $5 \times 50 \mathrm{~mm}^{2}$ was also used as the substrate. The curvature of the film/substrate composite was measured by the laser reflection method. The residual stress of the film was then calculated from the equilibrium equation of the bending plate [13]. A Jobin Yvon T64000 triple-Raman spectroscope was used to obtain the Raman spectra of the films. The spectral resolution was $1 \mathrm{~cm}^{-1}$, and the spectra were obtained in the range of $550-2500 \mathrm{~cm}^{-1}$.

In order to measure the strain, we prepared DLC free overhang by substrate etching technique. The DLC coated Si wafers, cleaved along the (011) direction, were etched in the diluted $\mathrm{KOH}$ solution $(5.6 \mathrm{~mol} / \mathrm{l})$ at $70{ }^{\circ} \mathrm{C}$ for $1-120 \mathrm{~min}$. In order to obtain a uniform etching condition, the solution was agitated during the etching process. Because of the anisotropic etching rate of $\mathrm{Si}$ in $\mathrm{KOH}$ solution, the (011) direction of the $\{111\}$ plane of the $\mathrm{Si}$ substrate was retained as an etching front. The DLC film is chemically so inert that we could not observe any surface damage or change in the film thickness after the etching process. The etched samples were wet cleaned in sequence using deionized water, methanol and acetone. The samples were then dried in ambient air to prevent any damage by blowing dry nitrogen. Fig. 1 shows the typical microstructure of DLC free overhang obtained by the present method. Because the mechanical constraint of the substrate was removed in the free overhang region, the length of the free overhang was recovered to its unstressed one of periodic sinusoidal shape. The etching depth was selected so that a fixed value of $A_{0} / \lambda$, where $A_{0}$ is the amplitude and $\lambda$ 
the wavelength of the free overhang, was observed regardless of the etching depth [8]. The sinusoidal edge extended more than 10 wavelengths without breaking the film. From a simple elastic analysis of thin film buckling [6,7], the biaxial elastic modulus, $E /(1-v)$, could be obtained by

$$
\frac{E}{1-v} \approx\left(\frac{\lambda}{\pi A_{0}}\right)^{2} \sigma_{0}
$$

where $E$ is the elastic modulus, $v$ Poisson's ratio, and $\sigma_{0}$ the residual compressive stress of the film. This equation shows that the biaxial elastic modulus can be obtained by measuring the amplitude and the wavelength of the free overhang for a film with a known residual compressive stress. The shape of the free overhang was observed by scanning electron microscope (SEM). SEM end-on view at a tilt angle of $80^{\circ}$ was used to measure the amplitude and wavelength of the sinusoidal edge.

\section{Results and discussion}

It is well known that high-energy ions play an important role to determine the structure and properties of DLC films. In the RF-PACVD process, the ion energy at the growth surface depends on both the time averaged cathode sheath potential by which the ion is accelerated to the growth surface, and inelastic collisions between ions and neutrals in the sheath. The latter is dependent on deposition pressure, and the former is approximately the same as the negative bias voltage of the cathode in a strongly asymmetric r.f. discharge system. For a precursor gas, the mean ion energy is thus known to be proportional to $V_{b} / \sqrt{P}$, where $V_{b}$ is the bias voltage of cathode and $P$ the deposition pressure [14]. In the present work, the measured properties of the film will be presented in terms of $V_{b} / \sqrt{P}$.

Fig. 2 shows the measured residual compressive stress of the film thicker than $1 \mu \mathrm{m}$ for various values of $V_{b} /$ $\sqrt{P}$. Because of high-energy ion bombardment on the growing surface, all DLC films have high residual compressive stress. In the present experimental condition, the residual stress was varied from 0.7 to $2.2 \mathrm{GPa}$ depending on the parameter, $V_{b} / \sqrt{P}$. The residual compressive stress shows a maximum at the value of $V_{b} /$ $\sqrt{P}$ of approximately $100 \mathrm{~V} / \mathrm{mtorr}^{1 / 2}$. The dependence of hardness on the parameter $V_{b} / \sqrt{P}$ showed the same behavior as that of the residual stress. The maximum hardness of $1950 \mathrm{kgf} / \mathrm{mm}^{2}$ was observed when the value of $V_{b} / \sqrt{P}$ was $100 \mathrm{~V} /$ mtorr $^{1 / 2}$. This behavior agrees with the previous work on the precursor gas effect in RF-PACVD [11]; dense diamond-like carbon film could be deposited only at an optimum ion energy. When the ion energy is smaller than the optimum one, polymeric film of high content of hydrogen is obtained. On the other hand, when the ion energy is too high,

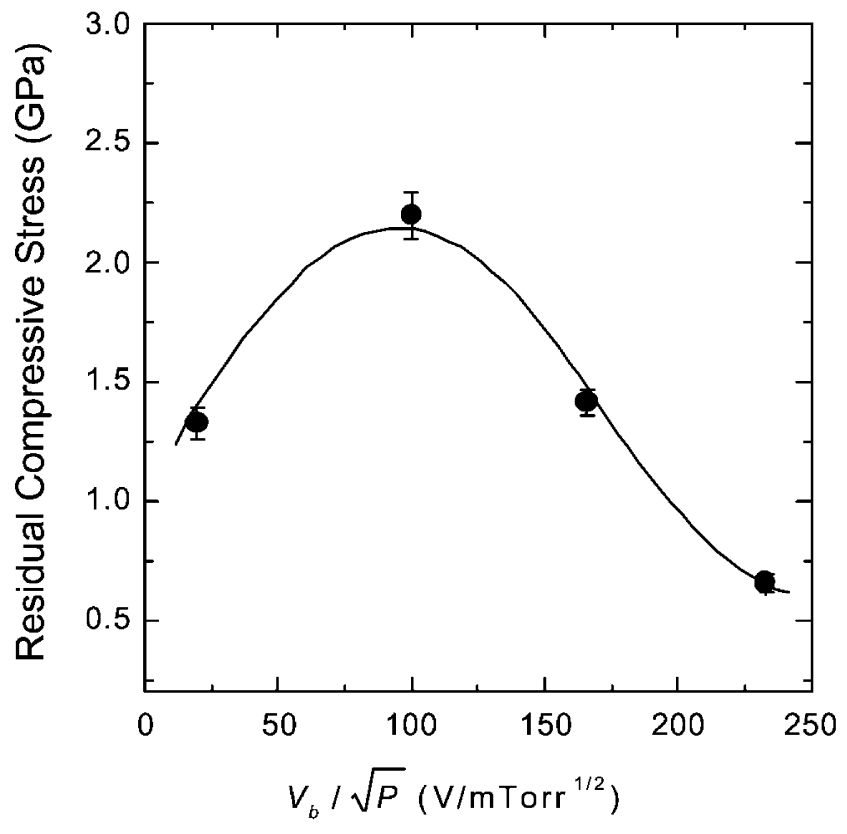

Fig. 2. Residual stress of the film thicker than $1 \mu \mathrm{m}$ for various values of $V_{b} / \sqrt{P}$.

thermal relaxation occurs during the film deposition process resulting in a film of greater graphitic composition. The observed changes in the residual stress and the hardness could be understood in terms of this structural variation according to the ion energy.

The structural change with the parameter $V_{b} / \sqrt{P}$ could also be observed by Raman spectroscopy. Fig. 3a shows typical Raman spectra for various values of $V_{b} / \sqrt{P}$ when the film thickness was approximately $1 \mu \mathrm{m}$. When the film was deposited at the value of $V_{b} / \sqrt{P}=20 \mathrm{~V} /$ mtorr $^{1 / 2}$, large tilted background caused by the luminescence of the polymeric structure was observed [15]. The background level was much reduced at the value of $V_{b} /$ $\sqrt{P}=100 \mathrm{~V} / \mathrm{mtorr}^{1 / 2}$. This spectral change shows that the film changes from polymeric to dense carbon film as the value of $V_{b} / \sqrt{P}$ increased from 20 to $100 \mathrm{~V} /$ mtorr $^{1 / 2}$. Further increasing $V_{b} / \sqrt{P}$ resulted in a more significant D-peak shoulder as can judged by the slope at approximately $1350 \mathrm{~cm}^{-1}$. Evolution of the D-peak is known to be due to the increase in the graphitic component [15]. The change in the shape of the Raman spectra qualitatively illustrates the structural change from polymeric to graphitic with increasing ion energy. More elaborate analysis includes deconvolution of the Raman spectrum with two Gaussian peaks: G-; and Dpeak [16]. It is empirically known that the G-peak position varied with changes in the atomic bond structure of the film. For example, graphitization of the film during high temperature annealing is correlated with the G-peak position shift to higher wave number [17]. Fig. $3 b$ shows the G-peak position of the film deposited in the present work. The G-peak position shifted to higher 

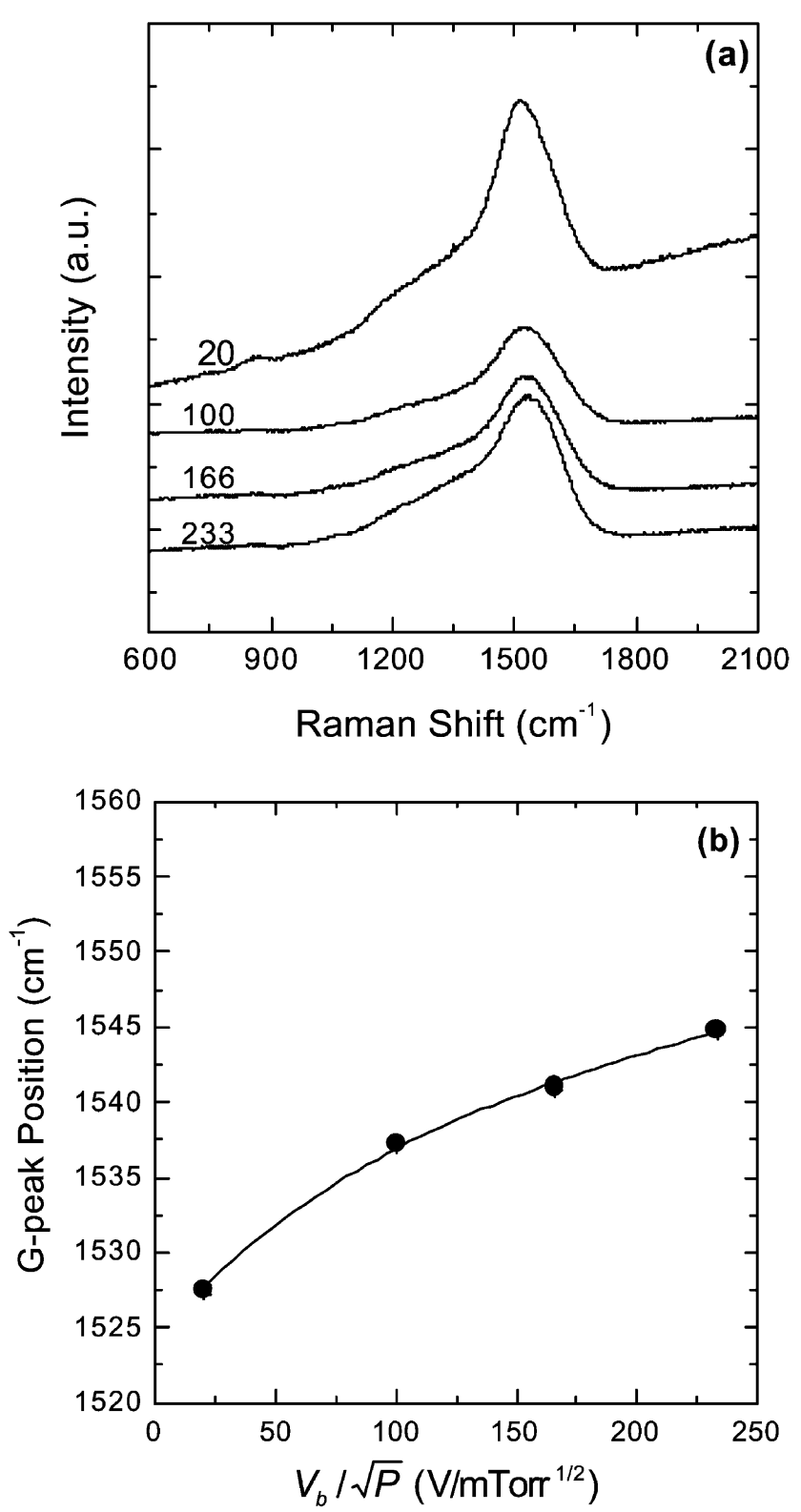

Fig. 3. (a) Raman spectra of the film with thickness of approximately $1 \mu \mathrm{m}$ for various values of $V_{b} / \sqrt{P}$. (b) G-peak position of the film with thickness of approximately $1 \mu \mathrm{m}$ for various values of $V_{b} / \sqrt{P}$.

wave number as the value of $V_{b} / \sqrt{P}$ increased, which is in agreement with the previous observation by Tamor et al. [18]. It can be thus said that the G-peak position shifts to higher wave number either when the graphitic component increased or when the polymeric component decreased.

Fig. 4 shows the dependence of the biaxial elastic modulus on the film thickness for various values of $V_{b} / \sqrt{P}$. The numbers on the data are the values of $V_{b} /$ $\sqrt{P}$ in unit of $\mathrm{V} / \mathrm{mtorr}^{1 / 2}$. When the value of $V_{b} / \sqrt{P}$ was $20 \mathrm{~V} / \mathrm{mtorr}^{1 / 2}$, the biaxial elastic modulus increased from 35 to $50 \mathrm{GPa}$ with increasing film thickness from 50 to $300 \mathrm{~nm}$. However, the biaxial elastic modulus saturated to $50 \mathrm{GPa}$ when the film thickness was larger than $300 \mathrm{~nm}$. Similar behavior was observed in the previous work where DLC film was deposited by RFPACVD using benzene as the precursor gas [8]; when the film thickness was smaller than $500 \mathrm{~nm}$, the smaller biaxial elastic modulus was observed, whereas a saturated value of the elastic modulus was obtained where the film was thicker than $500 \mathrm{~nm}$. In the previous work, the DLC film was deposited at the value of $V_{b} / \sqrt{P}=$ $126 \mathrm{~V} / \mathrm{mtorr}^{1 / 2}$ [8]. Even if this value of $V_{b} / \sqrt{P}$ is approximately six times higher than in the present work, the kinetic energy per carbon atom would be similar to that of the present work, because the ionized precursor in benzene has six carbon atoms whereas only one carbon atom exists in the methane precursor [11]. Therefore, these results show that the initial deposition layer has the lower elastic modulus when the ion energy was smaller than the optimum value. It was also reported in the previous work that the decrease in the elastic modulus of very thin film is not due to the interfacial reaction layer between the film and the substrate, but due to the structural evolution of the film during the initial stage of film deposition [8].

At the optimum value of $V_{b} / \sqrt{P}=100 \mathrm{~V} / \mathrm{mtorr}^{1 / 2}$ where the deposited film has the highest hardness and residual compressive stress, the effect of the film thickness on the biaxial elastic modulus, was much reduced. In the present range of the film thickness from 50 to $330 \mathrm{~nm}$, the biaxial elastic modulus of approximately $120 \mathrm{GPa}$ was obtained regardless of film thickness. On the other hand, when the values of $V_{b} / \sqrt{P}$ were larger than the optimum value, the biaxial elastic modulus

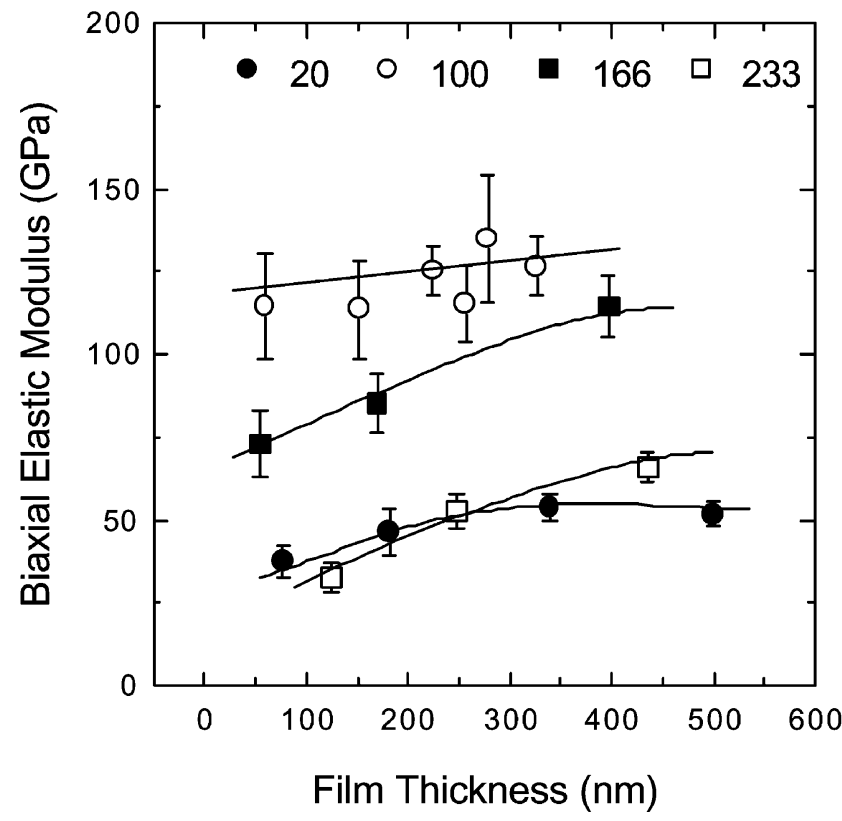

Fig. 4. Dependence of the biaxial elastic modulus on the film thickness for various values of $V_{b} / \sqrt{P}$. 


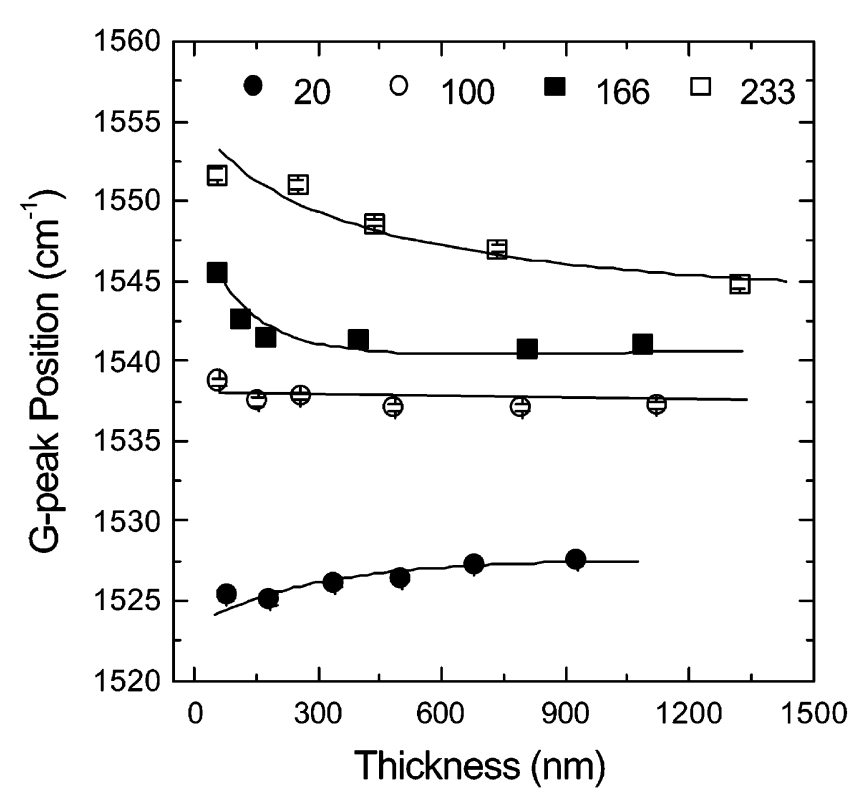

Fig. 5. Dependence of the G-peak position of Raman spectra on film thickness for various values of $V_{b} /{ } P$.

significantly decreased with decreasing film thickness. When the value of $V_{b} / \sqrt{P}$ was $166 \mathrm{~V} / \mathrm{mtorr}^{1 / 2}$, the biaxial elastic modulus decreased from 108 to $74 \mathrm{GPa}$ as the film thickness decreased from 420 to $50 \mathrm{~nm}$. Similarly, at the value of $V_{b} / \sqrt{P}=233 \mathrm{~V} / \mathrm{mtorr}^{1 / 2}$, the biaxial elastic modulus varied from 69 to $30 \mathrm{GPa}$ in the same thickness range. In contrast to the case of low $V_{b} /$ $\sqrt{P}$ values $\left(20 \mathrm{~V} / \mathrm{mtorr}^{1 / 2}\right)$, we could not observe the saturated behavior of the elastic modulus when the value of $V_{b} / \sqrt{P}$ was higher than $100 \mathrm{~V} / \mathrm{mtorr}^{1 / 2}$. It can be thus said that the initial deposition period of the graphitic film is more significant than in the polymeric film.

Fig. 5 shows the dependence of the G-peak position of the Raman spectra on film thickness for various values of $V_{b} / \sqrt{P}$. At the optimum ion energy where $V_{b} /$ $\sqrt{P}$ was $100 \mathrm{~V} /$ mtorr $^{1 / 2}$, the G-peak position did not vary with film thickness. This behavior can be compared with the constant biaxial elastic modulus regardless of film thickness as shown in Fig. 4. It can be thus said that at the optimum ion energy, the structure of the initial deposition layer is identical to that obtained in the later deposition stage. On the other hand, when the value of $V_{b} / \sqrt{P}$ was $20 \mathrm{~V} /$ mtorr $^{1 / 2}$, the G-peak position shifted to a lower wave number with decreasing film thickness. Because the polymeric component shifted the G-peak to lower wave number (see Fig. 3), this result shows that the initial deposition layer has higher content of the polymeric component. When the value of $V_{b} / \sqrt{P}$ was larger than $100 \mathrm{~V} /$ mtorr $^{1 / 2}$, however, the G-peak position shifted to higher wave number as the film thickness decreased, which shows that the more graphitic component was involved in the initial deposition layer. As in the elastic modulus change of Fig. 4, the depend- ence of the G-peak position on film thickness was more significant when the value of $V_{b} / \sqrt{P}$ was higher than $100 \mathrm{~V} / \mathrm{mtorr}^{1 / 2}$. This analysis result confirms that the initial deposition period of the graphitic film is more significant than in the polymeric film.

The present results showed that the structural evolution occurs when the ion energy used in the film deposition deviates from the optimum value for the dense and hard DLC film deposition. In these cases, the elastic modulus of the very thin film was smaller than that obtained in the thick film. However, the reason for the decrease in the elastic modulus for the very thin film was different according to the deposition condition. When the ion energy was smaller than the optimum value where the polymeric film was obtained, the initial deposition layer contained more polymeric component. In the opposite case where ion energy was higher than the optimum value, the initial deposition layer contained more graphitic component.

\section{Conclusions}

A recently suggested method for the measurement of thin film elastic modulus could provide a tool to characterize the mechanical properties of very thin films, since the substrate effect on the measurement could be completely excluded. The present work showed that the structural evolution during the initial stage of DLC film deposition could be investigated from the measurement of the elastic property as a function of film thickness. The structural evolution was investigated in the DLC films deposited by RF-PACVD. In both cases of lower and higher ion energies than the optimum value, decreases in the elastic modulus were observed in very thin films. Analysis of Raman spectra shows that changes in the elastic modulus is intimately related to structural evolution. In the polymeric films deposited when the ion energy was lower than the optimum value, the Gpeak shifted to lower wave number showing that the initial deposition layer has higher polymeric component. For higher ion energy than the optimum value, however, more graphitic film was deposited in the early stage of deposition, which made the G-peak position shift to higher wave number with decreasing film thickness. Only at the optimum ion energy, the structure and properties of very thin films are identical to those of thick films.

\section{Acknowledgments}

This work was financially supported by the Ministry of Science and Technology of Korea. Partial support from the Korea Science and Engineering Foundation through the Center for Advanced Plasma Surface Engineering at the Sungkyunkwan University is gratefully acknowledged. 


\section{References}

[1] W.C. Oliver, G.M. Pharr, J. Mater. Res. 7 (1992) 1564.

[2] T. Sawa, Y. Akiyama, A. Shinmamoto, K. Tanaka, J. Mater. Res. 14 (1999) 2228.

[3] P. Pastorelli, A.C. Ferrari, M.G. Beghi, C.E. Bottani, J. Robertson, Diamond Rel. Mater. 9 (2000) 825.

[4] Y. Kim, J. Electr. Mater. 26 (1997) 1002.

[5] D. Scheider, Th. Witke, Th. Schwarz, B. Schöneich, B. Schultrich, Surf. Coating Technol. 126 (2000) 136.

[6] S.-J. Cho, K.-R. Lee, K.Y. Eun, J.-H. Hahn, D.-H. Ko, Thin Solid Films 341 (1999) 207.

[7] S.-J. Cho, K.-R. Lee, K.Y. Eun, J.-H. Jeong, D. Kwon, Diamond Rel. Mater. 8 (1999) 1067.

[8] J.-W. Chung, C.-S. Lee, D.-H. Ko, J.-H. Hahn, K.Y. Eun, K.R. Lee, Diamond Rel. Mater. 10 (2001) 2069.
[9] J. Robertson, Surf. Coating Technol. 50 (1992) 185.

[10] B. Cord, J. Scherer, IEEE Trans. Mag. 36 (2000) 67.

[11] K.-R. Lee, Y.-J. Baik, K.Y. Eun, Diamond Rel. Mater. 3 (1994) 1230.

[12] K.-R. Lee, Y.-J. Baik, K.Y. Eun, Diamond Rel. Mater. 2 (1993) 218.

[13] K.-R. Lee, Y.-J. Baik, K.Y. Eun, MRS Proc. 308 (1993) 101.

[14] A. Bubenzer, B. Dischler, G. Brandt, P. Koidl, J. Appl. Phys. 54 (1983) 4590.

[15] M. Yoshikawa, Mater. Sci. Forum 52-53 (1989) 365.

[16] M. Yoshikawa, G. Katagiri, H. Ishida, T. Akamatsu, J. Appl. Phys. 64 (1988) 6464.

[17] D.R. Tallant, J.E. Parmeter, M.P. Siegal, R.L. Simpson, Diamond Rel. Mater. 4 (1995) 191.

[18] M.A. Tamor, W.C. Vassell, J. Appl. Phys. 76 (1994) 3823. 